\title{
Construction and testing of a plasmonic sensor using an amplifier system (one cavity and two rings) with two plasmonic waveguides
}

\author{
Hamid Abbasi \\ University of Mazandaran
}

\begin{abstract}
In this study, we seek to analyze and numerically evaluate a plasmonic sensor. To form the sensor structure, we use several amplifiers, such as two rings attached to each other and a cavity, as well as two metal insulating metal waveguides (MIM). At the beginning of this simulation, we must examine the resonant wavelengths and the refractive index of the resonators using the finite difference time domain method. By changing the refractive index and changing the dimensions of the cavity and the rims, we seek to investigate the sensor performance and the conduction characteristics of the plasmonics and to obtain the effect of these parameters. To evaluate the sensor performance, we calculate the three factors of sensitivity coefficient $S$, quality factor $Q$ and figure of merit (FOM), here we reach the sensitivity of $987.6 \mathrm{~nm} / R I U$. Such a plasmonic sensor with a simple framework and high optical resolution can be very useful for sensor systems on optical circuits.
\end{abstract}

Keywords: plasmonics; Surface plasmon polaritons; Metal-Insulator-Metal; refractive index sensor.

\section{Introduction}

By miniaturizing and reducing the dimensions of plasmonic structures, fully integrated highefficiency optical devices can be designed. Plasmonic science deals with the interaction of radiant electromagnetic waves on the surface of metals and their conducting electrons, and has the ability to enclose electromagnetic waves at dimensions much smaller than the radiant wavelength [1,2]. Plasmonics are divided into superficial plasmon plasmons and localized surface plasmons[3-5]. Plasmon surface polaritons (SPPs) are transverse electromagnetic waves bounded at the interface between metal and dielectric materials that can propagate up to several micrometers. The ability of surface plasmon polaritons to overcome the limitations of classical optical diffraction has made their use as energy and information carriers in fully integrated, popular and attractive circuits and optical devices. Among the various structures of Polariton, SPP surface plasmons, insulated metal-metal structures, and insulated metalinsulated structures are two common types of plasmonic structures. Also, the most important plasmonic components are based on the structure of active and inactive devices. Active environments are anisotropic and can be changed by applying an external factor, refractive index and length, but inactive environments are isotropic and have only one refractive index and are unsuitable for switches and sensors. Currently, one of the most important researches using surface plasmons is the detection of refractive index[6-12].. The resonance wavelength or angle of surface plasmons is very sensitive to environmental changes, and by changing one of the two, the range of surface plasmon effects can be controlled at the nanoscale, leading to a significant application in the development of high sensitivity. High integrity and design of refractive index sensors will be applied. In this research, we try to build a plasmonic sensor by using plasmonic structures and changing the refractive index and examining the resonance wavelength [13-15].

\section{Structural model and theory analysis}

The Droud model is used to describe metal and plasmonic waveguides in the design of sensors, as this model can be integrated into finite difference time domain simulations: (1) $(\omega)=\varepsilon_{\infty}-\omega_{\mathrm{p}}^{2} / \omega^{2}+i \gamma \omega$

Here $\varepsilon_{\infty}=1$ gives the medium constant for the infinite frequency, $\omega_{\mathrm{p}}=1.37 \times 1016$ refers to bulk frequency for plasma, $\gamma=3.21 \times 1013$ means damping frequency for electron oscillation, 
and $\omega$ shows incident light angular frequency. The proposed structure is shown in Figure 1, which consists of two waveguides and a cavity and two rings attached to each other. When the simulation starts, the input wave starts moving from the left side of the waveguides and goes to the right side of the waveguide after passing through the amplifiers. The width of the two waveguides is $\mathrm{w}_{1}=50 \mathrm{~nm}$. The cavity has a length of $\mathrm{w}_{2}=20 \mathrm{~nm}$ and a height of $100 \mathrm{~nm}$, which is located at a distance of $12 \mathrm{~nm}$ from the waveguide. The two rims attached to each other have an inner radius of $r=65 \mathrm{~nm}$ and an outer radius of $\mathrm{R}=100 \mathrm{~nm}$. $P_{\text {in }}$ and $P_{\text {out }}$ are the monitors for measuring the input wave and the output wave, respectively, and the transmission is calculated by $\mathrm{T}=\mathrm{P}_{\text {out }} / \mathrm{P}_{\text {in }}$.

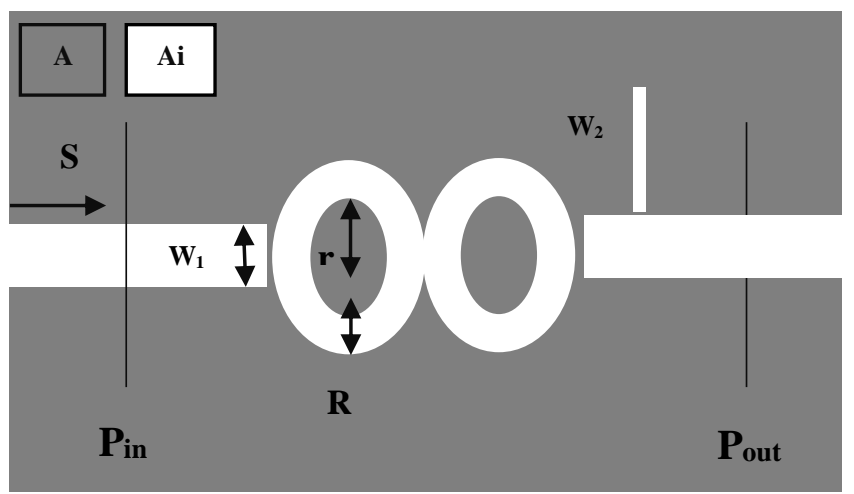

Figure 1: Two-dimensional image of a plasmonic sensor

The simulation bed is made of silver and the environment inside the rings, cavities and waveguides is composed of air. Because the wavelengths of the waveguides are smaller than the wavelength of the radiated light, only the TM core wave is present in the sensor structure. The TM wave, with a strong magnetic field intensity, starts to excite from the left and propagates in the waveguide, and the closer it gets to the output, the lower its intensity. Each resonators reflects part of the input wave to the input part. The distribution of the electric field at the resonant frequency of the simulated structure is shown in Figure 2. According to the shape, the maximum radiation takes place inside the rims. When the field distribution in the cavity and the two rings is the same, the energy loss is reduced. As a result, all dimensions must be optimized to achieve maximum field distribution in the rings and cavities.

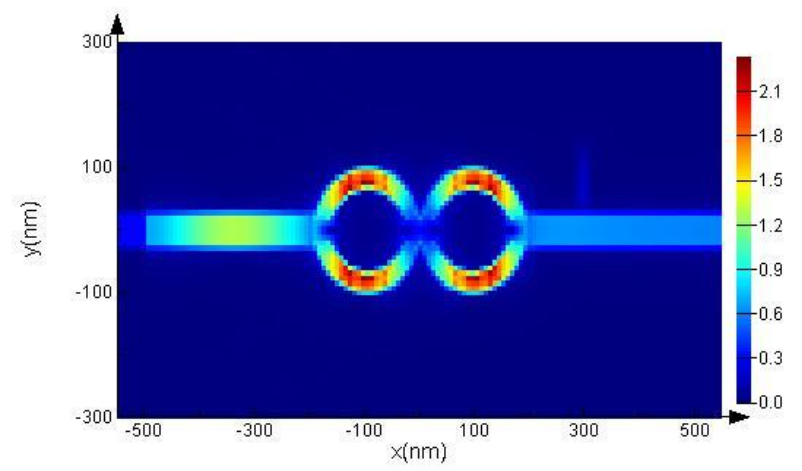

Figure 2: Electric field distribution at resonant frequency

\section{Fracture coefficient simulation and measurement methods}

We study the resonant behavior of the desired plasmonic sensor numerically and theoretically. For numerical analysis, we use the finite difference time domain simulation method with perfectly matched layer boundary conditions (PML). The reason for using this method is to 
reduce the numerical reflection. We consider the uniform mesh size for the $\mathrm{x}$ and $\mathrm{y}$ directions to be 8 and $8 \mathrm{~nm}$, respectively, and perform the simulation in two dimensions. The reason for this is to reduce the simulation time and achieve the desired result. The effective refractive index of the sensor with two rings attached to each other and a cavity in the wavelength range of 500 to $1500 \mathrm{~nm}$ is calculated and the transmission spectrum obtained from the sensor device is shown in Figure 3.

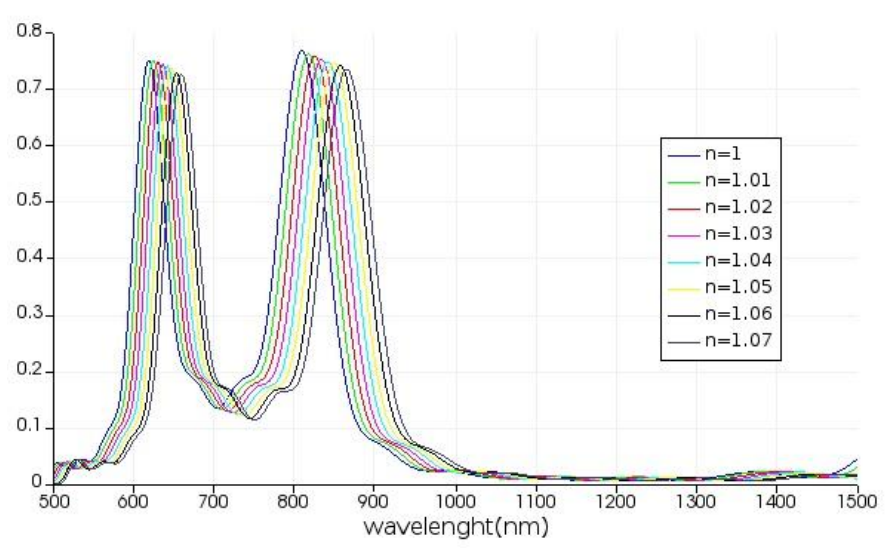

Figure 3: Transmission spectra of a plasmonic refractive index with two cavities

We increased the dielectric refractive index by a step of 0.01 from 1 to 1.07 , which resulted in a change in the resonance spectra and wavelengths. The first characteristic to be measured for a sensor is the $\mathrm{S}$ sensitivity, which is used to quantify the sensitivity of refractive index sensors: $\mathrm{S}=\Delta \lambda / \Delta \mathrm{n} \quad(\mathrm{nm} / \mathrm{RIU})$

In this equation, $\Delta \lambda$ is the change in resonance wavelength and $\Delta \mathrm{n}$ is the change in refractive index. According to Figure 3, the sensor transmission spectra have two peaks, which according to Figure 4 have the highest sensitivity for the refractive index $n=1.07$, which is equal to 987.6 $\mathrm{nm} / \mathrm{RIU}$, and the lowest value for the refractive index $\mathrm{n}=1.02$, which is equal to Is with 599.6 $\mathrm{nm} /$ RIU. According to this diagram, there is a relatively linear relationship between the two parameters of resonance wavelength and refractive index.

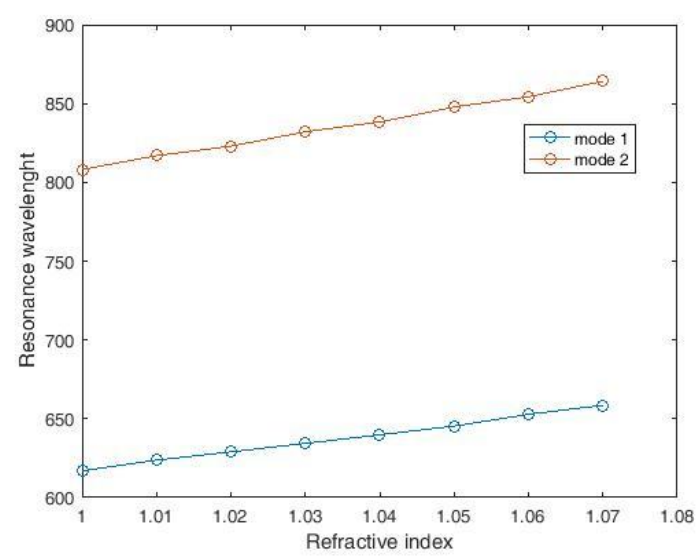

Figure 4: Resonance wavelength versus refractive index analysis

Since sensitivity alone is not a measure of good performance for comparing different types of sensors, and light resolution is also very important for sensors, high FOM is required to compare sensors: 
$\mathrm{FOM}=\mathrm{S} / \mathrm{FWHM}$

We also need the quality factor of the sensors:

$\mathrm{Q}=\lambda$ res / FWHM

Equations 2, 3, and 4 are factors that evaluate the performance of a plasmonic sensor. Using these three equations, we plot the sensitivity coefficient $S$, the quality coefficient $Q$ and the figure of merit (FOM). When the two rings are connected and the cavity is placed at a distance of $12 \mathrm{~nm}$ from the waveguide, we change their refractive index. As shown in Figure 5, mode 2 , which corresponds to the peak to the right of Figure 3, is more sensitive and mode 1 is less sensitive. Higher sensitivity reduces the figure of merit (FOM) at the desired point.

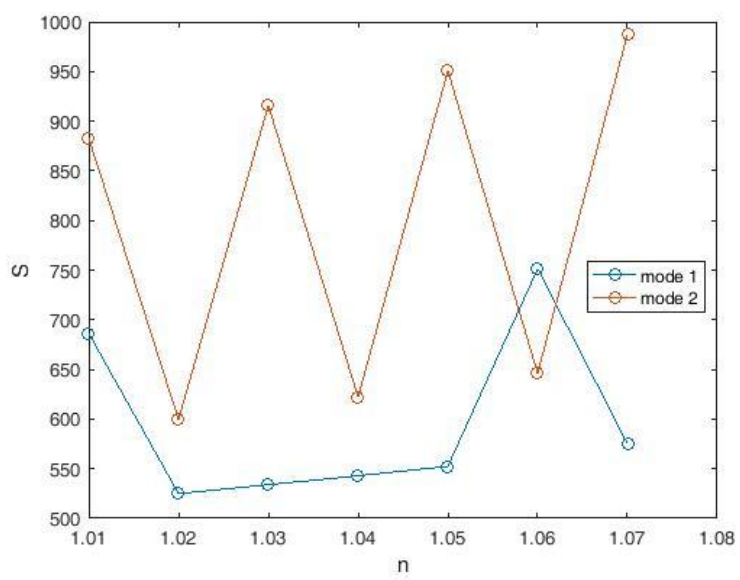

Figure 5: Plasmonic sensor sensitivity coefficient diagram

Increasing the length of the cavity and rims can improve the sensitivity performance of the sensor with a smaller FOM size, which may result in a longer light path and more energy loss.

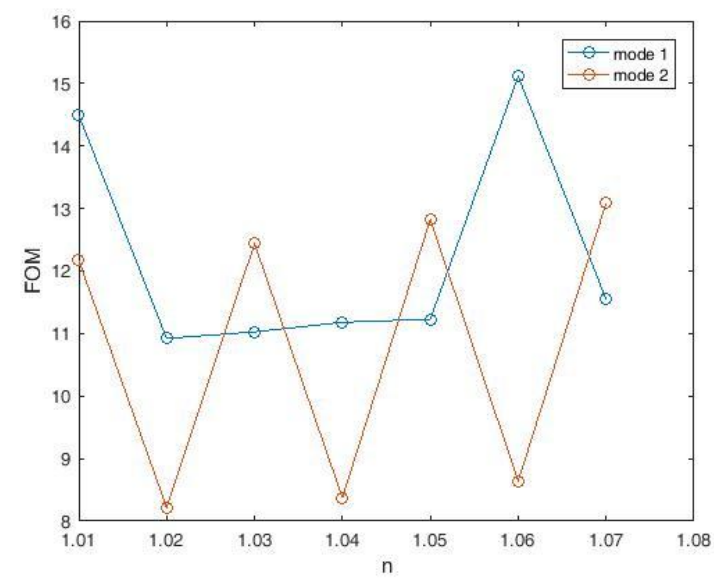

Figure 6: Plasmonic sensor FOM competency diagram 


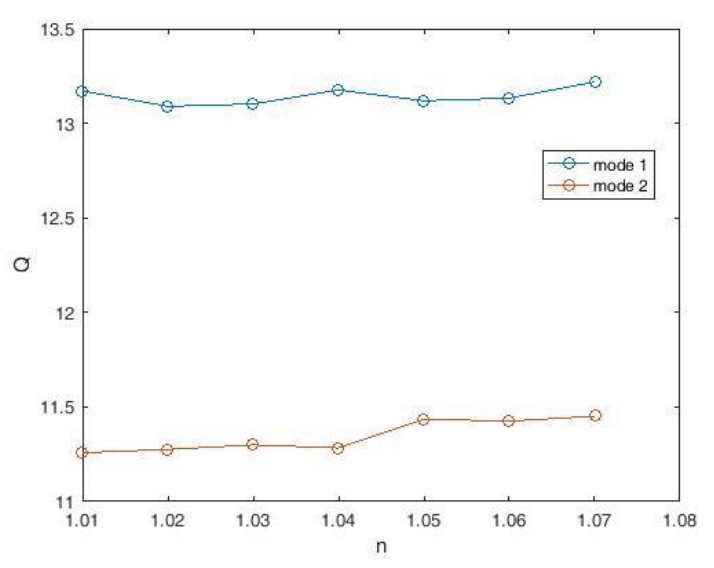

Figure 7: Plasmonic sensor quality coefficient diagram

According to Figure 7, we obtain the quality coefficient using Equation 4 and dividing the wavelength by FWHM, whose value in the refractive index $n=1.07$, which has the highest sensitivity coefficient, reaches 11.4520 .

\section{Conclusion}

Sensors based on surface plasmon resonance are attractive and popular due to the high surface sensitivity of surface plasmon polaritons. This sensor has received a lot of attention due to the fact that with a very small change in the refractive index of the structure, it causes a very large change in the propagation characteristics of the wave. Also as a sensor, it needs a high sensitivity factor (S) and a high competency digit (FOM) to provide excellent performance with high optical resolution. In this research, we have designed and simulated a plasmonic refractive index sensor using a cavity, two interconnected rings and two waveguides. For clarity of results and for better comparison, the refractive index of the structure changes from 1 to 1.07 and the resonant wavelength is calculated at each stage. The proposed sensor structure can be considered as a high-sensitivity nanosensor with a value of $987.6 \mathrm{~nm} / \mathrm{RIU}$, which due to its high resolution accuracy, can change the refractive index of 0.01 for materials with a refractive index between 1 and 07 / Is, recognize. Our results and analysis in this study, due to the small size of the structure configuration and high sensitivity, can lead to further development of plasmonic waveguides in the field of loss reduction and excellent light control in plasmonic structures.

Competing Interest: The authors declare no conflicts of interest.

Data availability. The data that support the findings of this study are available from the corresponding author upon reasonable request.

\section{References}

1.Cen, C.L .; Liu, L .; Zhang, Y.B .; Chen, X.F .; Zhou, Z.G .; Yi, Z .; Ye, X .; Tang, Y.J .; Yi, Y.G .; Xiao, S.Y. Tunable absorption enhancement in periodic elliptical hollow graphene arrays. Opt. Mater. Express 2019, 9, 706716.

2.Lin, H .; Ye, X .; Chen, X.F .; Zhou, Z.G .; Yi, Z .; Niu, G .; Yi, Y.G .; Hua, Y.T .; Hua, J.J .; Xiao, S.Y. Plasmonic absorption enhancement in grapheme circular and elliptical disk arrays. Mater. Res. Express 2019, 6,045807 .

3. Stefan A. Maier.(2007).Plasmonics: Fundamentals and Applications.

4.Ebbesen, Thomas W, A. Dereux, and C. W. Barnes. Surface plasmon subwavelength optics. Nature 2003 , 424.6950, 824-830. 
5.Wu, W. Ultra-high resolution filter and optical field modulator based on a surface plasmon polariton. Optics Letters 2016, 41.10, 2310.

6. Chen, L. Numerical analysis of a near-infrared plasmonic refractive index sensor with high figure of merit based on a fillet cavity. Optics Express 2016, 24.9, 9975.

6. Tong, L. Recent advances in plasmonic sensors. Sensors 2014, 14.5, 7959.

7. Y. Shen, J. H. Zhou, T. R. Liu, Y. T. Tao, R. B. Jiang, M. X. Liu, G. Xiao, J. Zhu, Z. K. Zhou, X. Wang, C. Jin, and J. Wang, Plasmonic gold mushroom arrays with refractive index sensing figures of merit approaching the theoretical limit. Nat. Commun 2013, 4, 2381.

8. Srivastava, Triranjita, R. Das, and R. Jha. Highly Sensitive Plasmonic Temperature Sensor Based on Photonic Crystal Surface Plasmon Waveguide. Plasmonics 2013, 8.2, 515-521.

9.Maisonneuve, M. Phase sensitive sensor on plasmonic nanograting structures. Optics Express 2011, 19.27, 26318-24.

10.Elsayed, Mohamed Y., Y. Ismail, and M. A. Swillam. Semiconductor plasmonic gas sensor using on-chip infrared spectroscopy. Applied Physics 2017, A 123.1, 113.

11.Yan, Shu Bin. A Refractive Index Sensor Based on a Metal-Insulator-Metal Waveguide-Coupled Ring Resonator. Sensors 2015, 15.11, 29183-91.

12.Huang, Ye Xiong. A plasmonic refractive index sensor based on a MIM waveguide with a side-coupled nanodisk resonator. IEEE, International Conference on Embedded and Real-Time Computing Systems and Applications IEEE 2014, 1-5.

13. Gomez-Cardona, N.; Reyes-Vera, E.; Torres, P. High Sensitivity Refractive Index Sensor Based on the Excitation of Long-Range Surface Plasmon Polaritons in H-Shaped Optical Fiber. Sensors 2020.

14. Yang X, Hua E, Su H, Guo J, Yan S. A Nanostructure with Defect Based on Fano Resonance for Application on Refractive-Index and Temperature Sensing. Sensors (Basel). 2020

15. Gryga, M.; Ciprian, D.; Hlubina, P. Bloch Surface Wave Resonance Based Sensors as an Alternative to Surface Plasmon Resonance Sensors. Sensors 2020 